\title{
Overcoming the Challenges of Splicing Dissimilar Diameter Solid-Core and Hollow-Core Photonic Band Gap Fibers
}

\author{
John P. Wooler, Seyed Reza Sandoghchi, David Gray, Francesco Poletti, Marco N. Petrovich, Natalie V. \\ Wheeler, Naveen Baddela and David J. Richardson \\ Optoelectronics Research Centre, University of Southampton, Southampton SO17 1BJ, United Kingdom \\ J.P.Wooler@soton.ac.uk
}

\begin{abstract}
The application of a novel splice technique for bonding solid core and hollow core microstructure fibers of dissimilar diameters, with low loss, is discussed and results of mechanical and optical performance presented.

OCIS codes: (060.4510) Optical communications; (230.3990) Microstructure devices; (060.0060) Fiber optics and optical communications; (220.0220) Optical design and fabrication; (230.0230) Optical devices
\end{abstract}

\section{The need to splice microstructured PBGF to solid-core fibers}

Air guiding hollow-core photonic band-gap fibers (HC-PBGF) offer the potential for ultralow nonlinearity, low latency and ultralow loss at $2 \mu \mathrm{m}$ and higher-order-mode (HOM) multiplexing. These traits are ripe for exploitation in future evolutions of communication systems [1] and while manufacturing techniques and optical performances develop at pace, there is an increasing need to splice them with more conventional all-solid fibers. Here we apply a splice strategy previously demonstrated for splicing together two HC-PBGFs [2] to the splicing of a HC-PBGF to a solid-core fiber, while working with dissimilar fiber diameters.

\section{The challenges to overcome and strategies}

Any splice technique must foremost manage the tendency for delicate microstructure (with struts around 100nm thick) and holey core to collapse when heat is applied [3]. A standard recipe for conventional singlemode fiber (SMF) splicing is typically too hot - the microstructure is destroyed and resulting splice loss is high (Fig 1, left). Reducing both arc current and duration can avoid significant collapse [4], though splices made in this way tend to be weak and brittle. The strategy we employ here involves a tack to fix the relative location of the fibers, a sweep of the join and finally a series of pulsed arcs over the splice to form a strong bond. This tack-sweep-pulse (TSP) strategy avoids damage to the microstructure by focusing heating at the surface creating a strong circumferential bond, while the average heating effect reaching the core is low, ultimately preserving the microstructure (Fig 1, center, left).
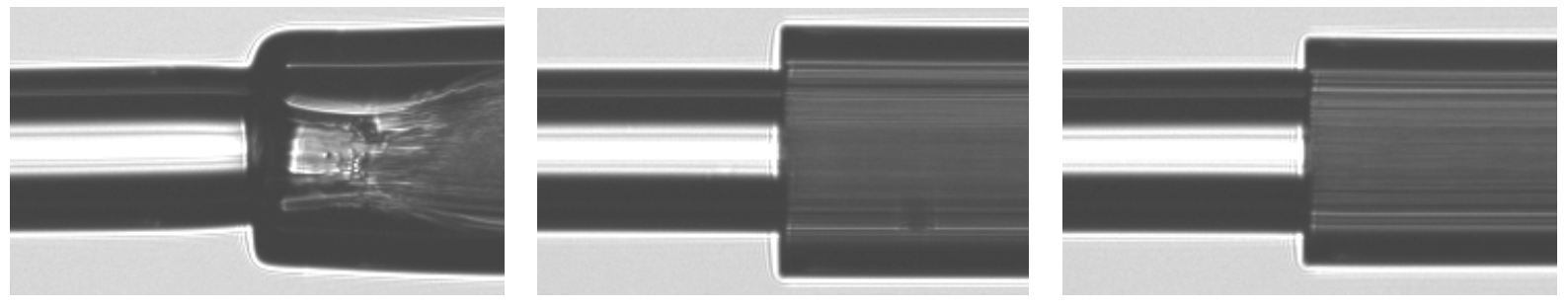

Fig. 1. Spliced $125 \mu \mathrm{m}$ solid core fiber - with SMF recipe to 37-cell (left), with TSP recipe: to 37-cell (center); to 19-cell (right)

Splicing of solid core and hollow core fibers with closely matched cladding diameters has previously been demonstrated, where angled cleaves at the splice were also used to minimize Fresnel reflections [7]. However, in some instances it may be preferable to fabricate HC-PBGFs with larger cladding diameters to best optimize some of their optical properties. In this work, for example, we optimize the splicing of a $125 \mu \mathrm{m}$ outer diameter solid core fiber with a $175 \mu \mathrm{m}$ outer diameter 19-cell HC-PBGF design [5] and with a $195 \mu \mathrm{m}$ 37-cell fiber [6]. The reduced bulk of solid glass to melt relative to the HC-PBGF is potentially an advantage. However, since the end face of the HC-PBGF is not fully protected from heat by the solid fiber, unless a TSP approach is adopted there is a greater risk of microstructure damage.

The core diameter in these 19-cell and 37-cell HC-PBGF [8] is also considerably larger than SMF $(\sim 26 \mu \mathrm{m}$ and $\sim 37 \mu \mathrm{m}$ ) and the consequent difference in mode-field diameter (MFD, $\sim 16 \mu \mathrm{m}$ and $\sim 26 \mu \mathrm{m}$ respectively) can lead to a significant mismatch loss (potentially $1.2 \mathrm{~dB}$ and $3.5 \mathrm{~dB}$ when butted to an SMF with $10.4 \mu \mathrm{m}$ MFD). Therefore here 
we favor a solid core partner fiber with a larger MFD than conventional SMF, where no core diffusion is required for low loss. A small residual MFD mismatch can be easily dealt with in an optimized splice to telecoms SMF.

The recipe we have developed yields a very neat join between hollow and solid fibers, and ultimately we achieve very robust splices which can be conveniently packed into splice protectors. The technique works equivalently well for 19-cell and 37-cell fibers despite their varying core and cladding diameters. This technique also copes well with dissimilar diameters, with solid fibers either larger or smaller than the HC-PBGF, although obviously the solid core fiber end face must by larger than the microstructured region.

\section{Characteristics when splicing solid core to HC-PBGF}

Micrographs of the end faces in a splice tensioned to failure clearly show the extent of the circumferential bond achievable with the splice strategy. An example with 37-cell fiber is shown in Fig 2, left, and the solid core fiber spliced to it seen in Fig 2, center. As a confirmation of the quality of the splices, and we are presently collecting statistical data, though provisionally losses are in the region $0.2-0.5 \mathrm{~dB}$.

HC-PBGF are multimode fibers, but good extinction between modes mean they can readily be operated singlemode if excited correctly - this excitation can be measured with the $S^{2}$ technique [9,10]. The amplitude of the peaks at various DGD values indicates the relative power in different high order modes (HOMs), We find that after the splice the transmitted power is still predominantly in the fundamental mode, with HOM extinction of $>32 \mathrm{~dB}$ for 19-cell and > 22dB in 37-cell. Here both fibers were spliced to a two-mode solid fiber with an LP01 MFD 16.4 $\mu \mathrm{m}$ and properly stripped to operate in a singlemode regime.
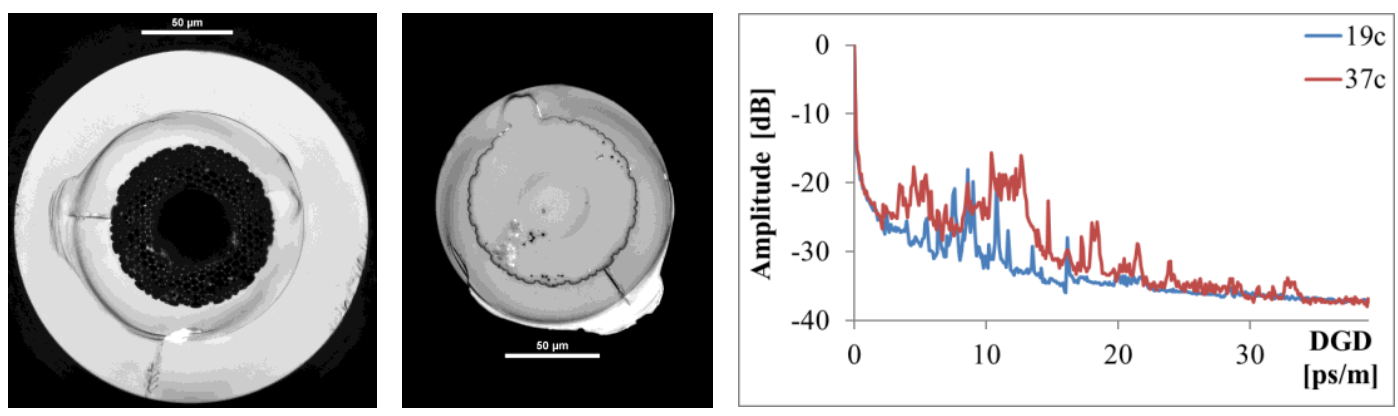

Fig. 2. End face images showing bonded regions in $195 \mu \mathrm{m}$ 37-cell HC-PBGF (left) and $125 \mu \mathrm{m}$ solid-core fiber (center) - $\mathrm{S}^{2}$ DGD values across spliced solid $125 \mu \mathrm{m}$ SMF and HC-PBGF (right)

\section{Conclusions}

We demonstrate effective splicing of dissimilar diameter solid-core fiber and HC-PBGFs together. The technique involves arc sweeping and rapid duty cycle to pulse power and prevent microstructure collapse. Low loss is expected when this technique is combined with well mode-matched fibers.

This work was supported by the EU 7th Framework Program under grant agreement 258033 (MODE-GAP) and by the UK EPSRC through grants EP/I01196X/1 (HYPERHIGHWAY) and EP/H02607X/1. Thanks to colleagues at OFS for providing the TMF sample.

\section{References}

[1] F. Poletti et al., Proc. OFC 2012, paper OTh1H.3

[2] J.P.Wooler, et al., Proc. OFC 2013, OM3I.5

[3] L. Xiao et al. , J. Light. Technol. 25 (11), 3563-3574, (2007)

[4] B. Bourliaguet, et al., Opt. Express, 11, 3412-17 (2003).

[5] N.V.Wheeler et al., Proc. OFC 2012, paper PDP5A.2 (Post deadline)

[6] Y.Jung, et al., Proc. OFC 2013, paper PDP5A.3 (Post deadline)

[7] F. Couny at al., IEEE Photonics Technology Letters, 19(13), 1020-1022 (2007)

[8] F. Poletti, et al., Nature Photonics, 7(4), 279 (2013)

[9] J. W. Nicholson et al., IEEE J. Sel. Top. Quantum Electron. 15, 61-70 (2009)

[10] D. Gray et al., Proc. ECOC 2012, paper Mo.2.F.1 